\title{
Effect of manufacturer-inserted mask leaks on ventilator performance
}

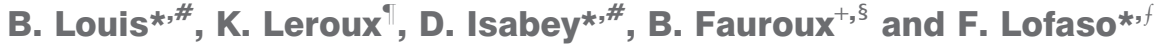

ABSTRACT: Most pressure-support devices use a single circuit with an exhalation port integrated in the mask. The aim of the current study was to compare the effects of masks having different manufacturer-inserted leaks on ventilator performance.

We simulated chronic obstructive pulmonary disease and restrictive disease. Four ventilators (VENTImotion (Weinmann, Hamburg, Germany), VPAP III STA (ResMed, Saint Priest, France), Synchrony 2 (Respironics, Nantes, France) and Vivo 40 (Breas, Saint Priest)) were tested with the recommended masks and with the masks having the largest and smallest leaks. Tests were performed with pressure support levels of 10,15 and $20 \mathrm{cmH}_{2} \mathrm{O}$. The in vivo evaluation compared two ventilators using recommended masks opposed in terms of exhaled port resistance. The ventilators were tested with their recommended mask, and after mask exchange.

The mask with the largest leak induced auto-triggering and/or increased inspiratory-trigger sensitivity was the VENTImotion under both simulated conditions and VPAP III STA under the simulated obstructive-disease condition. The mask with the smallest leak-increased inspiratorytrigger delay was Synchrony 2 in the simulated obstructive-disease condition and increased rebreathing. The in vivo study confirmed the bench results.

When switching to a mask that has a different leak, evaluation is needed to adjust trigger sensitivity and pressurisation level and to check the absence of rebreathing.

KEYWORDS: Interface, leaks, noninvasive ventilation, patient-ventilator synchrony, trigger

$\mathbf{N}$ oninvasive positive-pressure ventilation (NPPV) is recommended as the first-line treatment for acute and chronic respiratory failure [1]. Successful NPPV requires adaptation of mechanical ventilation to the patient's needs. If ventilation is ineffective or uncomfortable, the patient may have trouble adapting to the device and may even refuse NPPV $[1,2]$. The broad array of available ventilators and masks should increase the likelihood that a ventilatormask combination suited to the patient is found. Most ventilators, notably pressure-support (PS) devices, use a single circuit with an exhalation port located as close as possible to the patient's face, often within the mask itself [3, 4]. Many manufacturers validate the performances of their ventilators only with their own masks. Using a ventilator with a mask from a different manufacturer is therefore not recommended. However, a patient who is acclimatised to a specific ventilator may be more comfortable with a mask from a different manufacturer. In addition, patients on home ventilation have their ventilator settings determined at the hospital with a given mask but often subsequently switch to another mask at home.

The aims of the present study were to compare the leak levels of several masks and to evaluate the effects of masks having different leak levels on the performance of four ventilators.

\section{MATERIALS AND METHODS}

Experimental in vitro study

Evaluation of manufacturer-inserted mask leaks through different masks

The pressure/flow relationship of the mask exhalation port (leak) was evaluated by fitting the mask to a life-size mannequin. Compressed air was used to pressurise the mask to a range of predetermined values between 5 and $20 \mathrm{cmH}_{2} \mathrm{O}$. A pneumotachograph (Fleisch \#1; Gould Electronique, Longjumeau, France) coupled to a differential pressure transducer (Validyne DP45 \pm $3.5 \mathrm{cmH}_{2} \mathrm{O}$; Validyne, Northbridge, CA, USA) served to measure the airflow delivered to the mask. Another differential pressure transducer
AFFILIATIONS

*INSERM, Unit 955

\#Université Paris 12, Faculté de Médecine, Créteil,

-ADEP Assistance, Puteaux,

${ }^{+}$AP-HP Paediatric Pulmonary Dept, Hôpital Armand Trousseau,

${ }^{\S}$ Université Pierre et Marie CurieParis 6, INSERM UMR, Paris, and ${ }^{f}$ AP-HP, Raymond Poincaré Teaching Hospital, Université Versailles-St Quentin en Yvelines, Physiology Functional Testing, and Technological Innovations Centre, Garches, France.

\section{CORRESPONDENCE}

F. Lofaso

Service de Physiologie-Explorations Fonctionnelles

Hôpital Raymond Poincaré 92380 Garches

France

E-mail: f.lofaso@rpc.ap-hop-paris.fr

Received:

Nov 282008

Accepted after revision:

Aug 122009

First published online:

Aug 282009 
(Validyne DP45 $\pm 35 \mathrm{~cm} \mathrm{H} \mathrm{H}_{2} \mathrm{O}$ ) with one port connected to the mask and the other open to the atmosphere was used to measure the pressure inside the mask.

\section{Evaluation of the effect manufacturer-inserted mask leaks on ventilator performance}

A two-chamber Michigan test lung (MII Vent Aid TTL; Michigan Instrument, Grand Rapids, MI, USA) was used to evaluate the effect of manufacturer-inserted mask leaks on ventilator performance (fig. 1). To simulate a respiratory effort, the second chamber of the Michigan test lung (driving chamber) was connected to a flow-rate generator that could generate various waveforms previously stored in a microcomputer. The two chambers were physically connected to each other by a small metal component that allowed the driving chamber to lift the testing chamber. The flow-rate generator, developed by our laboratory as previously described, was built by associating pressurised air, flow-rate measurement, and a servo valve driven by a microcomputer [5]. An adult-sized mannequin head was connected to the lung chamber via a circuit with a dead space of $120 \mathrm{~mL}$. Two adultsized mannequin heads were available to easily adapt the masks without additional leaks between the mask and the mannequin. Flow $\left(V^{\prime}\right)$ was measured between the mannequin head and the lung chamber using a pneumotachograph (Fleish $\mathrm{n}^{\circ}$ 2, Lausanne, Switzerland) connected to a differential pressure transducer (Validyne DP $45 \pm 3.5 \mathrm{cmH}_{2} \mathrm{O}$ ). Pressure at the level of the mask ( $P$ aw $)$ was measured via a differential pressure transducer (Validyne DP $45 \pm 56 \mathrm{cmH}_{2} \mathrm{O}$ ). A second pneumotachograph was inserted between the mask and the ventilator to measure the leak through the mask exhalation port $\left(V^{\prime}\right.$ leak $=V^{\prime}$ c $-V^{\prime}$ where $V^{\prime}$ c: flow delivered by the ventilator and $V^{\prime}$ leak is leak through the mask exhalation port; fig. 1) and the volume of air that returned into the ventilator circuit during expiration. Signals were digitised at $200 \mathrm{~Hz}$ by an analogical/digital system (MP100, Biopac Systems, Goleta, CA, USA) and recorded on a microcomputer for further analysis. Each mask was carefully fitted on the mannequin head and before each recording we systematically checked that $30 \mathrm{cmH}_{2} \mathrm{O}$ of positive pressure, did not induce leak measurable by the pneumotachograph when the exhalation port and the connection to the lung chamber were closed. To obtain this result mask strips were very tight round the mannequin head, which could not be done in an in vivo condition. In addition we retrospectively checked that instantaneous leak $\left(V^{\prime}\right.$ leak $\left.=V^{\prime} \mathrm{c}-V^{\prime}\right)$ corresponded to the expected leak in accordance with $P$ aw.

The compliance of the test chambers and the resistance (a parabolic airway resistor) (Pneuflo ${ }_{\circledR}$ Airway resistor Rp20 and Rp5; Michigan Instrument, Grand Rapids, MI, USA) were set at $100 \mathrm{~mL} \cdot \mathrm{cmH}_{2} \mathrm{O}^{-1}$ and $\mathrm{Rp} 20$, respectively, to simulate obstructive disease and at $30 \mathrm{~mL} \cdot \mathrm{cmH}_{2} \mathrm{O}^{-1}$ and $\mathrm{Rp} 5$, respectively, to simulate restrictive disease. Respiratory rate was set at 10 breaths $\cdot \mathrm{min}^{-1}$ for the obstructive-disease simulation and 20 breaths $\cdot \min ^{-1}$ for the restrictive-disease simulation. During both simulations, spontaneous tidal volume $(V \mathrm{~T})$ was $300 \mathrm{~mL}$ and the ratio of inspiratory time (tI) over total respiratory-cycle duration (ttot) was 33\%. The inspiratory flow contour was rounded to simulate a physiological flow contour.

Positive end-expiratory pressure (PEEP) was $4 \mathrm{cmH}_{2} \mathrm{O}$. Three levels of PS were evaluated: 10, 15, and $20 \mathrm{cmH}_{2} \mathrm{O}$, these corresponded to an inspiratory positive airway pressure (IPAP) of 14, 19 and $24 \mathrm{cmH}_{2} \mathrm{O}$, respectively. Whenever possible, the inspiratory trigger was set at the most sensitive

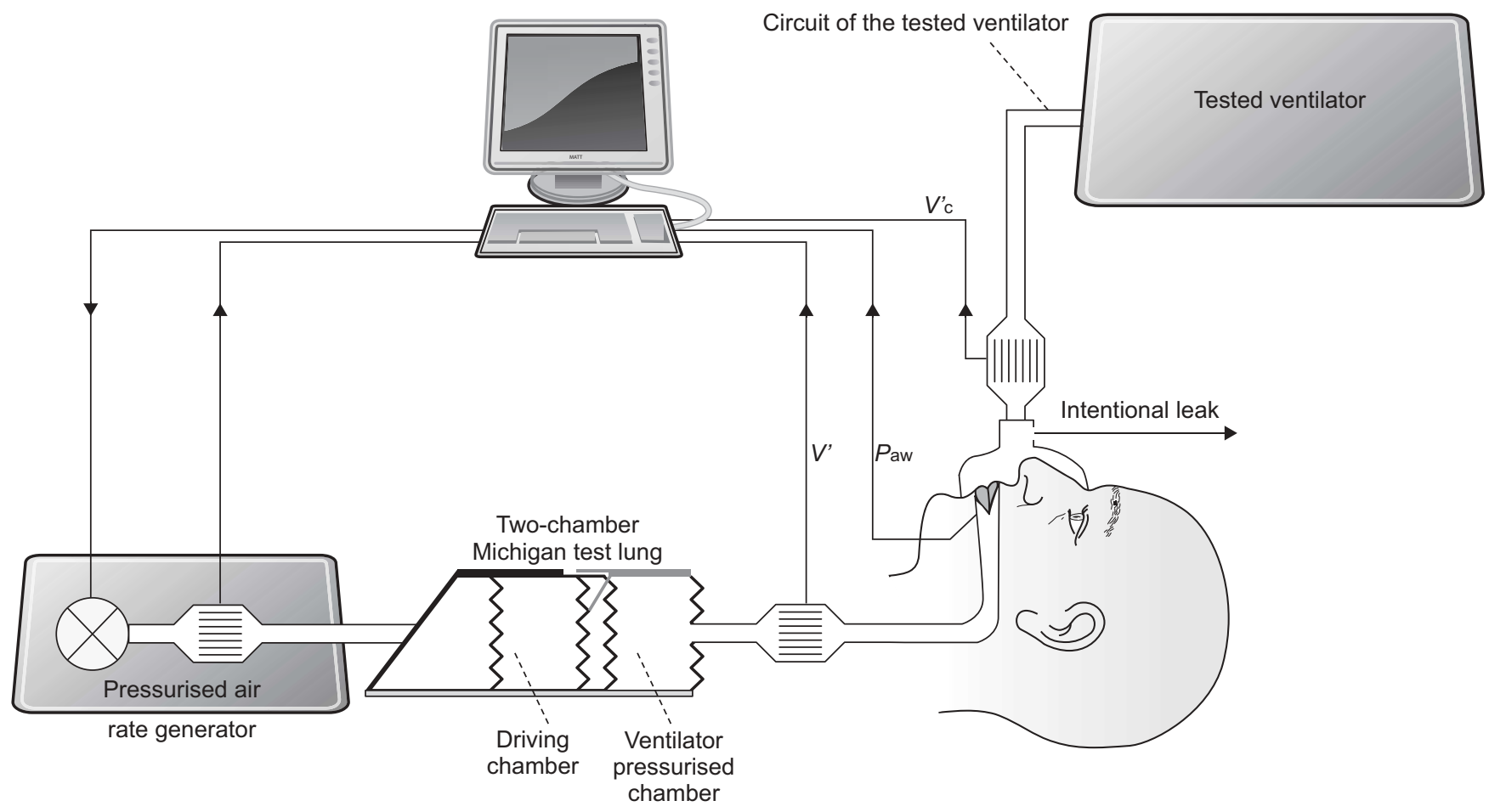

FIGURE 1. Lung bench test. $V^{\prime}$ : flow into the lung chamber; $V^{\prime} c$ : flow delivered by the ventilator; Paw: airway pressure. 


\section{TABLE 1 The 10 masks tested}

\begin{tabular}{lc} 
Brand & Model \\
\hline Nasal masks & SOMNOplus \\
Weinmann (Hamburg, Germany) & Ultra Mirage \\
ResMed (Saint Priest, France; North Ryde, Australia) & Mirage Activa Large \\
ResMed (Saint Priest, France; North Ryde, Australia) & Profile Lite \\
Respironics (Nantes, France; Murrysville, PA, USA) & Flexifit 407 \\
Fisher \& Paykel (Villebon-s/Yvette, France) & IQ 50293 \\
Breas (Saint Priest, France; Mölnlycke, Sweden) & Ultra Mirage Full Face Medium \\
Face masks & Comfort Full \\
ResMed (Saint Priest, France; North Ryde, Australia) & Flexifit 432 \\
Respironics (Nantes, France; Murrysville, PA, USA) & JOYCE Full Face \\
Fisher \& Paykel (Villebon-S/Yvette, France) &
\end{tabular}

level that did not induce auto-triggering, whereas the expiratory trigger was set at the most sensitive level associated with a minimum $t \mathrm{I}$ of $1 \mathrm{~s}$.

As previously described [5], the following parameters were computed from each pressure and/or flow trace: inspiratory trigger sensitivity, based on the trigger time $(\Delta t)$, i.e. the time from inspiration onset to achievement of an airway pressure greater than the PEEP level. Pressurisation performance (PP) was evaluated as the mean pressure above the PEEP level during the first $0.5 \mathrm{~s}$ of inspiration [6]. This mean pressure was expressed as a percentage of the PS level delivered by the ventilator. $t \mathrm{I}$ was calculated from the flow signal and $V \mathrm{~T}$ (the volume delivered to the lung chamber) by integrating the flow signal. Re-breathing was evaluated as the percentage of the expired volume remaining in the circuit at the end of expiration $[4,7]$.

\section{Devices}

The relationship between the leak level and airway pressure was measured for six nasal masks and four face masks (table 1). The choice of the ventilator was based on the ventilator specification which advised the use of a specific mask from the same manufacturer. Four ventilators were tested (table 2) with their recommended masks and with the masks having the largest and the smallest leak levels. Evaluation with nonrecommended masks were performed without changing the ventilator settings; if auto-triggering occurred, a second evaluation was done after setting the inspiratory trigger at the most sensitive level not associated with auto-triggering and the expiratory trigger at the most sensitive value associated with a minimum $t \mathrm{I}$ of $1 \mathrm{~s}$.

\section{Experimental in vivo study}

Devices tested

Two ventilators were tested: the one with the recommended mask that had the most resistive exhaled port and the one with the recommended mask that had the less resistive exhaled port. The aim was to test two the ventilators with their recommended mask, and thereafter to exchange the masks.

\section{Subjects}

This study was approved by the local ethics committee and written informed consent was obtained for all subjects. Four healthy subjects aged 36-51 yrs were included.

\section{Methods}

Two pneumotachographs (Fleisch \#2), each connected to a differential pressure transducer (Validyne MP $45 ; \pm 3 \mathrm{cmH}_{2} \mathrm{O}$ ), were used (fig. 2). One pneumotachograph was inserted upstream to the mask exhalation port whereas the other was inserted downstream to the mask exhalation port. The first pneumotachograph upstream of the mask was fixed to a mannequin face (fig. 2). The air entered through the mannequin nostrils into a short tube (length $20 \mathrm{~cm}$, internal diameter $0.75 \mathrm{~cm}$, total resistance at $1 \mathrm{~L} \cdot \mathrm{s}^{-1} 6.8 \mathrm{cmH} \mathrm{C}_{2} \mathrm{O} \cdot \mathrm{L}^{-1} \cdot \mathrm{s}^{-1}$ ) which was connected to the pneumotachograph; and the second pneumotachograph was connected to the subject via a mouth piece while the subject wore a nose clip to be sure no leak occurred between the subject and the interface. This additional

TABLE 2 The four ventilators tested

VENTImotion
VPAP III STA UM Cfg
Synchrony 2
Vivo 40




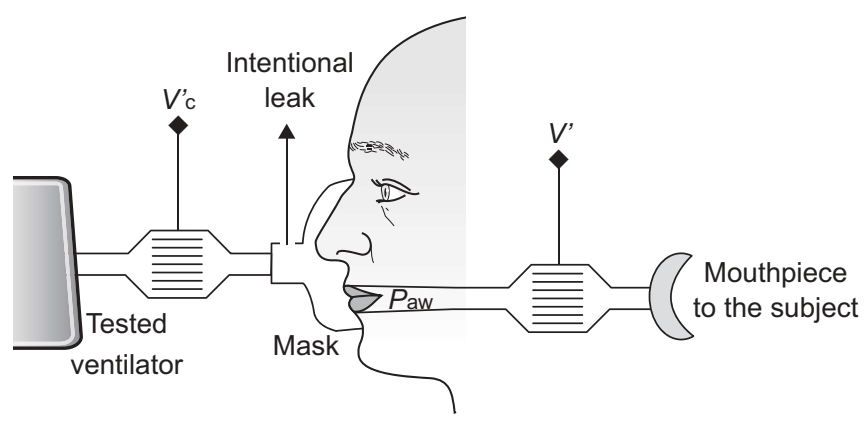

FIGURE 2. The in vivo experimental set up. $V^{\prime}$ : flow into the lung chamber; $V^{\prime}{ }^{\prime}$ : flow delivered by the ventilator; Paw: airway pressure.

circuit induced a dead space increase of $45 \mathrm{~mL}$. Paw was measured using a differential pressure transducer (Validyne MP 45 model; $\pm 100 \mathrm{cmH}_{2} \mathrm{O}$ ) connected to the mask (fig. 2). All signal outputs were digitised at $200 \mathrm{~Hz}$ (MP100, Biopac Systems) and recorded.

\section{Experimental protocol}

With each device, adjustments were performed with the/a recommended mask. Pressure support was adjusted with the study participant sitting comfortably. The backup rate was set at the lowest rate. PEEP was set at $4 \mathrm{cmH}_{2} \mathrm{O}$. The IPAP level, the slope of pressure support and the expiratory trigger were adjusted according to the preference of the subject. The inspiratory trigger was set at the most sensitive level that did not induce autotriggering. The study participants were blinded to the condition. This initial condition was maintained to obtain $10 \mathrm{~min}$ of stabilisation followed by $3 \mathrm{~min}$ of recording. Thereafter, the recommended mask was changed for the nonrecommended mask without changing the settings of the ventilator. This new condition was maintained to obtain $10 \mathrm{~min}$ of stabilisation followed by $3 \mathrm{~min}$ of recording.

\section{Data analysis}

The recordings were analysed breath by breath. The parameters analysed were the same as during the experimental in vitro study.

\section{Statistics of the in vitro and the in vivo studies}

Each parameter was averaged for 30 respiratory cycles. Data were given as mean $\pm \mathrm{SD}$. For the in vitro study the effect of the mask change was evaluated using a paired t-test. The level of significance was set at $5 \%$. Because the number of subjects tested in the in vivo study was too small, the comparison was only qualitative.

\section{RESULTS}

\section{Experimental in vitro study}

The pressure-flow relationships of the mask ports are shown in figure 3. The characteristics of the masks were very similar, with the exception of the Weinman SOMNOplus, whose resistance was higher, and the ResMed Ultra Mirage Full Face Medium, whose resistance was lower. These two masks were therefore tested with each of the four ventilators.

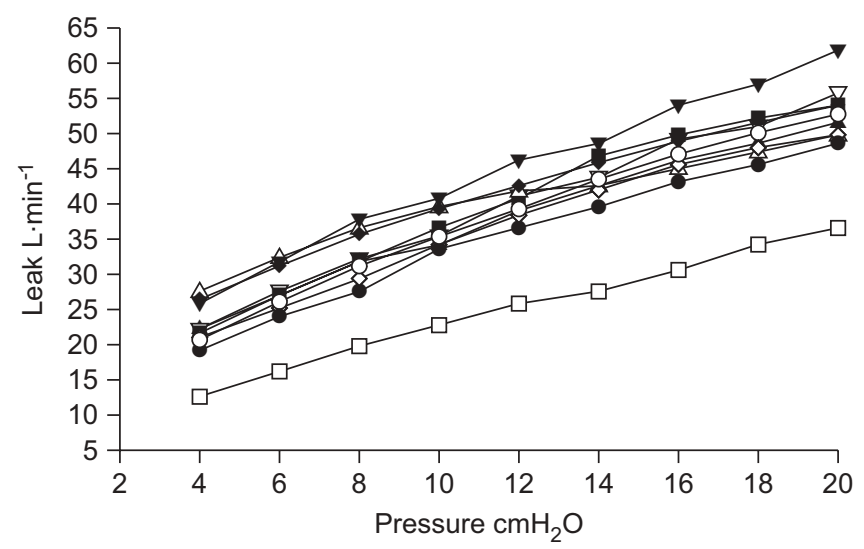

FIGURE 3. Pressure-flow relationships of the exhalation ports of the 10 masks. $\square$ : SOMMOplus (Weinmann, Hamburg, Germany); $\mathbf{\Delta}$ : Comfort Full (Respironics, Nantes, France); $\triangle$ : Mirage Activa Large (ResMed, Saint Priest, France); $\nabla: I Q$ 50293 (Breas, Saint Priest, France); $\mathbf{\square}$ : Flexifit 407 (Fisher \& Paykel, Villebon-s/ Yvette, France); $\mathbf{\nabla}$ : Ultra Mirage Full Face (ResMed, Saint Priest, France); $\diamond:$ Ultra Mirage (ResMed, Saint Priest, France); - Profile Lite (Respironics, Nantes, France); : Flexifit 432 (Fisher \& Paykel, Villebon-s/Yvette, France); ○: JOYCE Full Face (Weinmann, Hamburg, Germany).

\section{Performance of the ventilators with their recommended masks}

The performance characteristics of the four ventilators tested with the recommended masks are reported in table 3 . We found major differences across ventilators regarding inspiratory-trigger sensitivity, $t \mathrm{I}$, and $V \mathrm{~T}$. Synchronisation between the simulated patient profile and the ventilator was possible except with the Vivo 40 ventilator under the restrictive-disease condition at IPAP $\geqslant 19 \mathrm{cmH}_{2} \mathrm{O}$.

\section{Effects on ventilator performance of the mask with the largest intentional leak}

The performance characteristics of the ventilators with the mask having the largest manufacturer-inserted mask leak (MIML) are shown in table 4. Auto-triggering occurred with the VENTImotion device in both simulated conditions and with the VPAP III STA device under the obstructive disease condition with the highest IPAP level. In addition, inspiratorytrigger sensitivity increased with the VPAP III STA in the obstructive-disease simulation when IPAP was 14 or $19 \mathrm{cmH}_{2} \mathrm{O}$. This increase in inspiratory-trigger sensitivity was associated with an increase in PP. The performance of the Synchrony was not noticeably changed when using the highleak mask. In contrast, performance of the Vivo 40 was substantially affected, with differences between the two simulated conditions and across PS levels and with either a decrease or an increase in inspiratory-trigger sensitivity.

Adjustment of the inspiratory trigger corrected the autotriggering observed with the VPAP III STA and VENTImotion under the obstructive-disease condition. After this adjustment, inspiratory-trigger sensitivity was better than with the recommended mask (table 5). In contrast, inspiratory-trigger adjustment corrected the auto-triggering observed with the VENTImotion under the restrictive-disease condition only when IPAP was $14 \mathrm{cmH}_{2} \mathrm{O}$. Ventilator-setting adjustment did not avoid the auto-triggering observed with Vivo 40 under the restrictive-disease condition when IPAP was $\geqslant 19 \mathrm{cmH}_{2} \mathrm{O}$. 
TABLE 3 Performance of the ventilators with the recommended masks

\begin{tabular}{|c|c|c|c|c|c|c|c|c|c|}
\hline Ventilator & Patient profile & $\begin{array}{c}\mathrm{PS} \\
\mathrm{cmH}_{2} \mathrm{O}\end{array}$ & $\begin{array}{l}\text { PEEP } \\
\mathrm{cmH}_{2} \mathrm{O}\end{array}$ & $\begin{array}{l}\text { IPAP } \\
\mathrm{cmH}_{2} \mathrm{O}\end{array}$ & $\Delta t \mathrm{~ms}$ & $t ı s$ & $V_{T} \mathbf{m L}$ & $\begin{array}{c}\text { Rebreating } \\
\% \text { of } V_{T}\end{array}$ & PP \% \\
\hline \multirow[t]{4}{*}{ VENTImotion } & Obstructive disease & 10 & $4.0 \pm 0.02$ & $14.9 \pm 0.04$ & $629 \pm 106$ & $1.6 \pm 0.03$ & $330 \pm 7$ & $0 \pm 0$ & $0.0 \pm 0.1$ \\
\hline & & 15 & $4.0 \pm 0.01$ & $19.7 \pm 0.04$ & $618 \pm 42$ & $1.6 \pm 0.04$ & $375 \pm 6$ & $0 \pm 0$ & $0.0 \pm 0.1$ \\
\hline & & 15 & $4.0 \pm 0.02$ & $20.3 \pm 0.08$ & $218 \pm 7$ & $1.1 \pm 0.01$ & $471 \pm 1$ & $17.2 \pm 0.3$ & $26.2 \pm 1.4$ \\
\hline & & 20 & $4.0 \pm 0.02$ & $25.5 \pm 0.11$ & $218 \pm 7$ & $1.0 \pm 0.59$ & $575 \pm 117$ & $22.5 \pm 5$ & $20.3 \pm 1$ \\
\hline \multirow[t]{4}{*}{ VPAP III STA UM Cfg } & Obstructive disease & 10 & $3.7 \pm 0.01$ & $13.5 \pm 0.02$ & $230 \pm 16$ & $2.3 \pm 0.02$ & $478 \pm 2$ & $0 \pm 0$ & $31.1 \pm 3.2$ \\
\hline & & 15 & $3.7 \pm 0.01$ & $18.3 \pm 0.02$ & $214 \pm 19$ & $2.3 \pm 0.02$ & $617 \pm 2$ & $0 \pm 0$ & $32.1 \pm 3.7$ \\
\hline & & 20 & $3.7 \pm 0.01$ & $23.0 \pm 0.04$ & $248 \pm 12$ & $2.4 \pm 0.01$ & $754 \pm 2$ & $0 \pm 0$ & $24.0 \pm 2.5$ \\
\hline & Restrictive disease & 10 & $3.6 \pm 0.02$ & $13.8 \pm 0.36$ & $112 \pm 5$ & $1.0 \pm 0.02$ & $392 \pm 6$ & $0 \pm 0$ & $52.2 \pm 2.6$ \\
\hline \multirow{5}{*}{ Synchrony 2} & & 15 & $3.8 \pm 0.13$ & $18.7 \pm 0.08$ & $274 \pm 82$ & $2.4 \pm 0.44$ & $659 \pm 122$ & $0 \pm 0$ & $27.4 \pm 13.7$ \\
\hline & & 20 & $3.7 \pm 0.02$ & $23.6 \pm 0.10$ & $248 \pm 25$ & $2.7 \pm 0.20$ & $828 \pm 63$ & $0 \pm 0$ & $28.6 \pm 8.1$ \\
\hline & Restrictive disease & 10 & $3.8 \pm 0.01$ & $15.9 \pm 0.05$ & $136 \pm 3$ & $1.0 \pm 0.01$ & $402 \pm 1$ & $0 \pm 0$ & $46.0 \pm 0.7$ \\
\hline & & 15 & $3.8 \pm 0.03$ & $20.6 \pm 0.06$ & $139 \pm 30$ & $1.0 \pm 0.01$ & $450 \pm 1$ & $0 \pm 0$ & $48.8 \pm 0.7$ \\
\hline & & 20 & $3.9 \pm 0.01$ & $25.4 \pm 0.09$ & $139 \pm 35$ & $1.0 \pm 0.01$ & $497 \pm 1$ & $0 \pm 0$ & $48.7 \pm 1$ \\
\hline \multirow[t]{6}{*}{ Vivo 40} & Obstructive disease & 10 & $3.7 \pm 0.02$ & $12.2 \pm 0.17$ & $402 \pm 46$ & $1.9 \pm 0.08$ & $386 \pm 13$ & $0 \pm 0$ & $1.9 \pm 2.6$ \\
\hline & & 15 & $3.7 \pm 0.02$ & $16.6 \pm 0.10$ & $342 \pm 69$ & $2.4 \pm 0.06$ & $558 \pm 15$ & $0 \pm 0$ & $4.4 \pm 2.1$ \\
\hline & & 20 & $3.7 \pm 0.07$ & $20.8 \pm 0.19$ & $381 \pm 88$ & $2.5 \pm 0.17$ & $658 \pm 47$ & $0 \pm 0$ & $2.7 \pm 2.4$ \\
\hline & Restrictive disease & 10 & $3.7 \pm 0.03$ & $14.6 \pm 0.14$ & $217 \pm 24$ & $1.0 \pm 0.01$ & $389 \pm 2$ & $0 \pm 0$ & $19.2 \pm 4.5$ \\
\hline & & 15 & AT & AT & AT & AT & AT & AT & AT \\
\hline & & 20 & AT & AT & AT & AT & AT & AT & AT \\
\hline
\end{tabular}

Data are presented as mean $\pm S D$, unless otherwise stated. PS: pressure support; PEEP: positive end-expiratory pressure; IPAP: inspiratory positive airway pressure measured; $t l$ : inspiratory time; $V T$ : tidal volume; rebreathing: percentage of expired volume remaining in the device circuit at the end of expiration; $\Delta t$ : time from inspiration onset to airway pressure above PEEP; PP: pressurisation performance; AT: auto-triggering. VENTImotion: Weinmann, Hamburg, Germany; VPAP III STA UM Cfg: ResMed, Saint Priest, France; Synchrony 2: Respironics, Nantes, France; Vivo 40: Breas, Saint Priest, France.

Effects on ventilator performance of the mask with the smallest intentional leak

Use of the mask with the smallest MIML either increased or decreased inspiratory trigger sensitivity, $t \mathrm{I}, V \mathrm{~T}$, and $\mathrm{PP}$ (table 6). Under the restrictive-disease condition with a high PS level, this mask was associated with increased rebreathing (table 6). Auto-triggering occurred with Synchrony 2 and the highest PS level in the obstructive-disease condition. Ventilator-setting adjustment was not possible, since trigger setting was automatic. In contrast, this mask corrected the auto-triggering observed with Vivo 40 in the restrictive-disease condition after ventilator-setting adjustment.

\section{Experimental in vivo study}

The ventilators and masks chosen for the in vivo study were first, the VENTImotion with its SOMNOplus mask, considering that this mask had the most resistive exhalation port, and second the VPAP III STA because this device can set with the "ResMed Ultra Mirage Full Face" Mask which had the less resistive exhalation port.

The results obtained in the four subjects with these two ventilators and their recommended masks are presented in table 7. As previously observed in the in vitro study, when the level of pressure support was similar in both conditions, VPAP III STA was associated with a longer $t \mathrm{I}$, a higher $V \mathrm{~T}$, a shorter $\Delta t$ and higher PP than the VENTImotion. In addition, rebreathing was observed in two subjects with the VENTImotion.

The results obtained in the four subjects with these two ventilators but after the mask change (which was not recommended by the manufacturers) are presented in table 8 . We observed that, similar to the in vitro study, auto-triggering occurred with VENTImotion when the leak was increased using the ResMed Ultra Mirage Full Face mask and rebreathing occurred in three subjects with the VPAP III STA when the leak was decreased using the SOMNOplus mask.

\section{DISCUSSION}

This bench study showed substantial differences in MIML across masks that are widely used in clinical practice and established that leak level affects ventilator performance. The mask with the largest leak was associated with auto-triggering and/or decreased inspiratory-trigger sensitivity with three of the four ventilators. The mask with the smallest intentional 


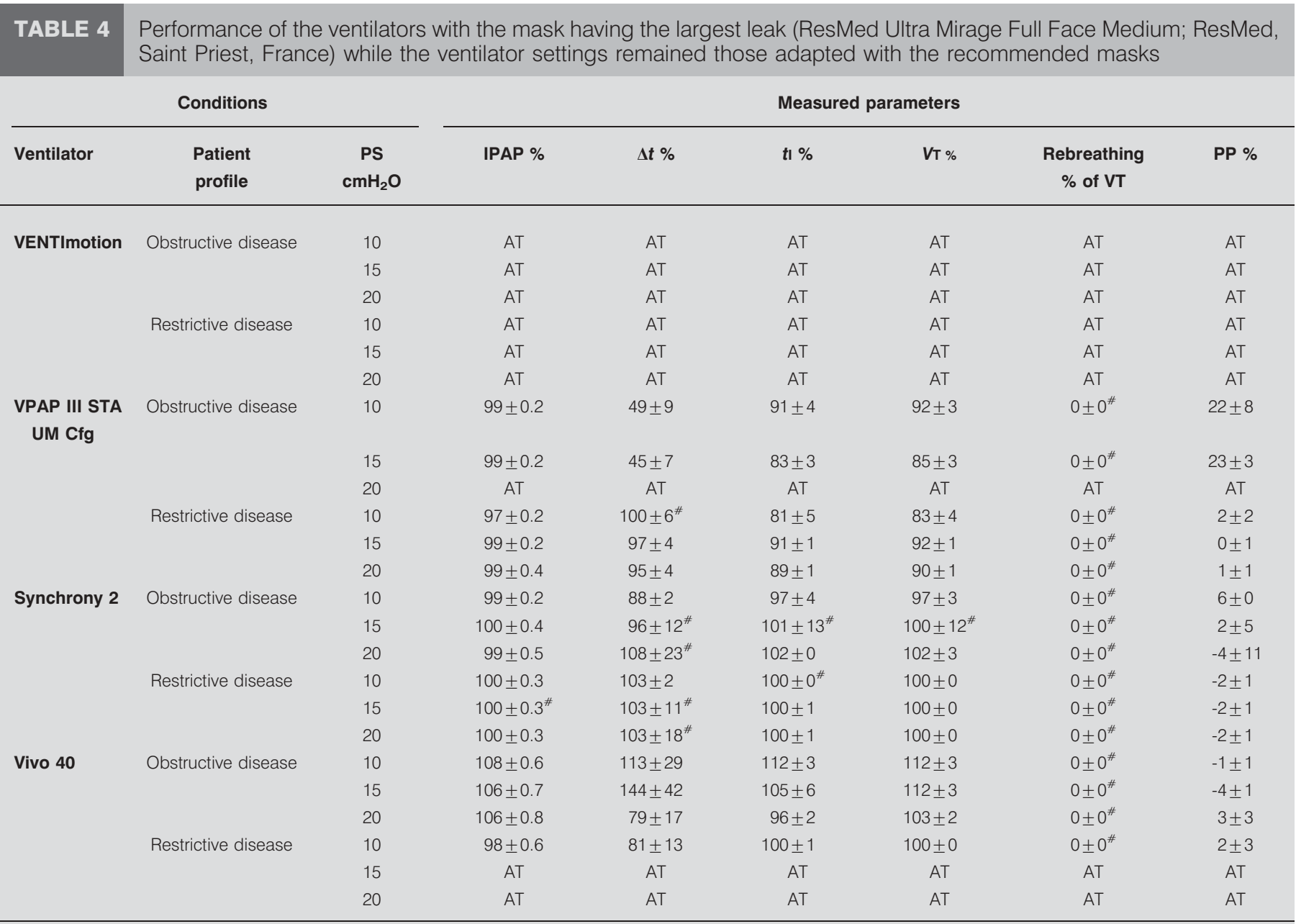

Data are presented as mean $\pm \mathrm{SD}$, unless otherwise stated. Note that the ResMed Ultra Mirage Full Face Medium is recommended for VPAP III STA, but this ventilator remained configured with the Ultra Mirage, which is therefore not recommended. Values are percentage of baseline value with the recommended mask (table 3), except for rebreathing, which is the percentage of tidal volume $(\mathrm{VT})$ and pressurisation performance (PP), which is the percentage change versus baseline. PS: pressure support; IPAP: inspiratory positive airway pressure measured; $\Delta t$ : time from inspiration onset to airway pressure above positive end-expiratory pressure; $t$ : inspiratory time; rebreathing: percentage of expired volume remaining in the device circuit at the end of expiration; AT: auto-triggering. VENTImotion: Weinmann, Hamburg, Germany; VPAP III STA UM Cfg: ResMed; Synchrony 2: Respironics, Nantes, France; Vivo 40: Breas, Saint Priest. \#: not statistically different from the baseline values.

leak was associated with increased rebreathing. An in vivo evaluation confirmed these results.

Although it was not the aim of the study, striking differences were observed between the ventilator devices. For some ventilators and in certain conditions the inspiratory trigger delay was inacceptable. It was previously considered that an acceptable inspiratory trigger needs to have a $\Delta t$ of $<150 \mathrm{~ms}$. None of the ventilators reached this value when simulating obstructive disease and only two ventilators reached this value when simulating restrictive disease. The systematic higher $\Delta t$ when simulating obstructive disease than when simulating restrictive disease was mainly explained by the fact that for the same negative pressure inside the testing chamber, produced by the driving chamber, at the beginning of the inspiration, the depression at the mask level was less when simulating obstructive disease than when simulating restrictive disease. This may be explained by the presence of a higher resistance (RP20 versus RP5) between the testing chamber and the mask which impedes the transmission of the negative pressure to the mask.

The other important difference between ventilators is the large difference in $V \mathrm{~T}$ despite a similar level of pressure support. This was explained by differences in the $t \mathrm{I}$ which was mainly influenced by the difference in expiratory trigger sensitivity between ventilators. For these reasons it is not recommended to change a pressure support device for another device without complete adjustment of the ventilator settings for the patient.

Importantly, the effect of the MIML level on the inspiratory trigger is variable and unpredictable. In one study, increasing the leak either induced auto-triggering or had no effect on the inspiratory trigger, depending on the type of ventilator [8] Similarly, a leak $>10 \mathrm{~L} \cdot \mathrm{min}^{-1}$ was associated with autotriggering in seven of eight ventilators and with effects on inspiratory-trigger time consisting in an increase (four ventilators) a decrease (one ventilator), or no change (two 


\begin{tabular}{|c|c|c|c|c|c|c|c|c|}
\hline TABLE 5 & $\begin{array}{l}\text { Performance of th } \\
\text { Saint Priest, Franc } \\
\text { occurred when ve }\end{array}$ & $\begin{array}{l}\text { entilato } \\
\text { once th } \\
\text { lator se }\end{array}$ & $\begin{array}{l}\text { the mas } \\
\text { ilator set } \\
\text { were tho }\end{array}$ & $\begin{array}{l}\text { ig the la } \\
\text { lere adj } \\
\text { apted } w\end{array}$ & $\begin{array}{l}\text { eak (Re } \\
\text { vith this } \\
\text { recomr }\end{array}$ & $\begin{array}{l}\text { Ultra Mir } \\
\text { in order } \\
\text { d masks }\end{array}$ & $\begin{array}{l}\text { ull Face Me } \\
\text { /erse auto-tri }\end{array}$ & $\begin{array}{l}\text { ResMed, } \\
\text { g, which }\end{array}$ \\
\hline Ventilator & Patient profile & $\begin{array}{c}\text { PS } \\
\mathrm{cmH}_{2} \mathrm{O}\end{array}$ & IPAP $\%$ & $\Delta t \%$ & $t 1 \%$ & VT \% & $\begin{array}{c}\text { Rebreathing } \\
\% \text { of } V_{T}\end{array}$ & PP $\%$ \\
\hline \multirow{5}{*}{ VENTImotion } & & 15 & $88 \pm 0.2$ & $37 \pm 3$ & $96 \pm 7$ & $115 \pm 11$ & $0 \pm 0^{\#}$ & $27 \pm 4$ \\
\hline & & 20 & $88 \pm 0.3$ & $38 \pm 5$ & $89 \pm 8$ & $107 \pm 12$ & $0 \pm 0^{\#}$ & $21 \pm 4$ \\
\hline & Restrictive disease & 10 & $85 \pm 0.3$ & $121 \pm 4$ & $93 \pm 7$ & $43 \pm 5$ & $0 \pm 0^{\#}$ & $-8 \pm 4$ \\
\hline & & 15 & AT & AT & AT & AT & AT & AT \\
\hline & & 20 & AT & AT & AT & AT & AT & AT \\
\hline
\end{tabular}

Data are presented as mean $\pm \mathrm{SD}$, unless otherwise stated. Values are percentage of baseline value with the recommended mask (table 3), except for rebreathing, which is the percentage of tidal volume $(\mathrm{VT})$ and pressurisation performance (PP), which is the percentage change versus baseline. PS: pressure support; IPAP: inspiratory positive airway pressure measured; $\Delta t$ : time from inspiration onset to airway pressure above positive end-expiratory pressure; $t$ : inspiratory time; rebreathing: percentage of expired volume remaining in the device circuit at the end of expiration; AT: auto-triggering. VENTImotion: Weinmann, Hamburg, Germany; VPAP III STA UM Cfg: ResMed. *: not statistically different from the baseline values.

ventilators) [9]. We also found that the inspiratory trigger was not consistently affected by the leak level. Some of these discrepancies may be ascribable to the experimental conditions. For example, one study found no effect of leakage on the inspiratory trigger [10], whereas another, conducted on the same ventilators, showed that a large leak induced autotriggering when the inspiratory trigger was excessively sensitive [11]. From a mechanical point of view, the effect of a leak on the inspiratory trigger cannot be predicted without detailed information on the algorithm used to drive ventilator behaviour, and particularly the ventilator whose aim is to maintain a constant PEEP as long as it detects no new patient effort. This behaviour is different if we assume that the ventilator acts as a perfect flow generator or a perfect pressure generator. In the first case, a decrease in the leak level facilitates the detection of the inspiratory effort by the ventilator, while this is not true in the second case (for more explanation see the online supplementary material).

Similarly, the effect of the leak on the expiratory trigger is unpredictable without detailed information on the ventilator software. Usually, the expiratory trigger is based on detection of the time when the flow rate reaches a given percentage of the peak inspiratory flow. The presence of a leak ( $V^{\prime}$ leak) may partly mask the fall in the patient's inspiratory flow $\left(\mathrm{x} \% \cdot V^{\prime} \neq \mathrm{x} \%\left(V^{\prime}+V^{\prime}\right.\right.$ leak $)$. Underestimation of $V^{\prime}$ leak should theoretically delay the expiratory trigger time, whereas overestimation should shorten the expiratory trigger time. Therefore, underestimation of the leak should increase $t$ I and $V \mathrm{~T}$, whereas overestimation should decrease both parameters. Thus, although the level of the leak is determined by the mask, estimation of this leak and therefore of the variations in $t \mathrm{I}$ and $V \mathrm{~T}$ is determined by the ventilator.

Our findings may appear to differ from a recent study on the effects of various masks on the performance of a range of ventilators [12]. However, these apparent discrepancies may be ascribable to differences in the study ventilators. Indeed, in the other study, the ventilator whose performance was the most affected by the mask with the largest leak in our study was not tested [12]. The experimental conditions were also different. Respiratory system compliance was $\leqslant 30 \mathrm{~mL} \cdot \mathrm{cmH}_{2} \mathrm{O}^{-1}$ in the other study [12] and $\geqslant 30 \mathrm{~mL} \cdot \mathrm{cmH}_{2} \mathrm{O}^{-1}$ in our study. In our study, the effects of mask changes were greatest under the obstructive-disease condition with a high respiratory-system compliance $\left(100 \mathrm{~mL} \cdot \mathrm{cmH}_{2} \mathrm{O}^{-1}\right)$. In addition, at a similar level of PS, $V$ T measured in the other study [12] was higher than in our study, although respiratory compliance was lower, suggesting that the simulated patient effort was higher. Nevertheless, increasing the leak affected $V \mathrm{~T}$ in both studies. We also showed that inspiratory-trigger performance was affected by the mask having the largest leak and that rebreathing was affected by the mask having the smallest intentional leak.

Our index of rebreathing $[4,7]$ did not exactly measure the rebreathing volume. In fact, by inserting a pneumotachograph between the mask with its exhalation port and the ventilator, we were able to measure the expiratory gas which returned and remained upstream of the exhalation port just before the next inspiration. Obviously this value overestimated the rebreathing, since a part of this volume is washed through the exhalation port and toward the atmosphere during inspiration. Nevertheless, ideally, the totality of expiratory gas should be washed through the exhalation port at the end of expiration. This means that $V \mathrm{~T} / \mathrm{tE}$ should be lower than the leak during expiration. When regarding the breathing pattern of patients under pressure support in the literature, the mean $V \mathrm{~T} / \mathrm{tE}$ was equal to $243 \mathrm{~mL} \cdot \mathrm{s}^{-1}\left(14.5 \mathrm{~L} \cdot \mathrm{min}^{-1}\right)$ during sleep in stable neuromuscular patients [13], equal to $333 \mathrm{~mL} \cdot \mathrm{s}^{-1}$ $\left(20 \mathrm{~L} \cdot \mathrm{min}^{-1}\right)$ during wakefulness in both neuromuscular patients and chronic obstructive pulmonary disease patients [14], and equal to $370 \mathrm{~mL} \cdot \mathrm{s}^{-1}\left(22 \mathrm{~L} \cdot \mathrm{min}^{-1}\right)$ in intubated patients, 


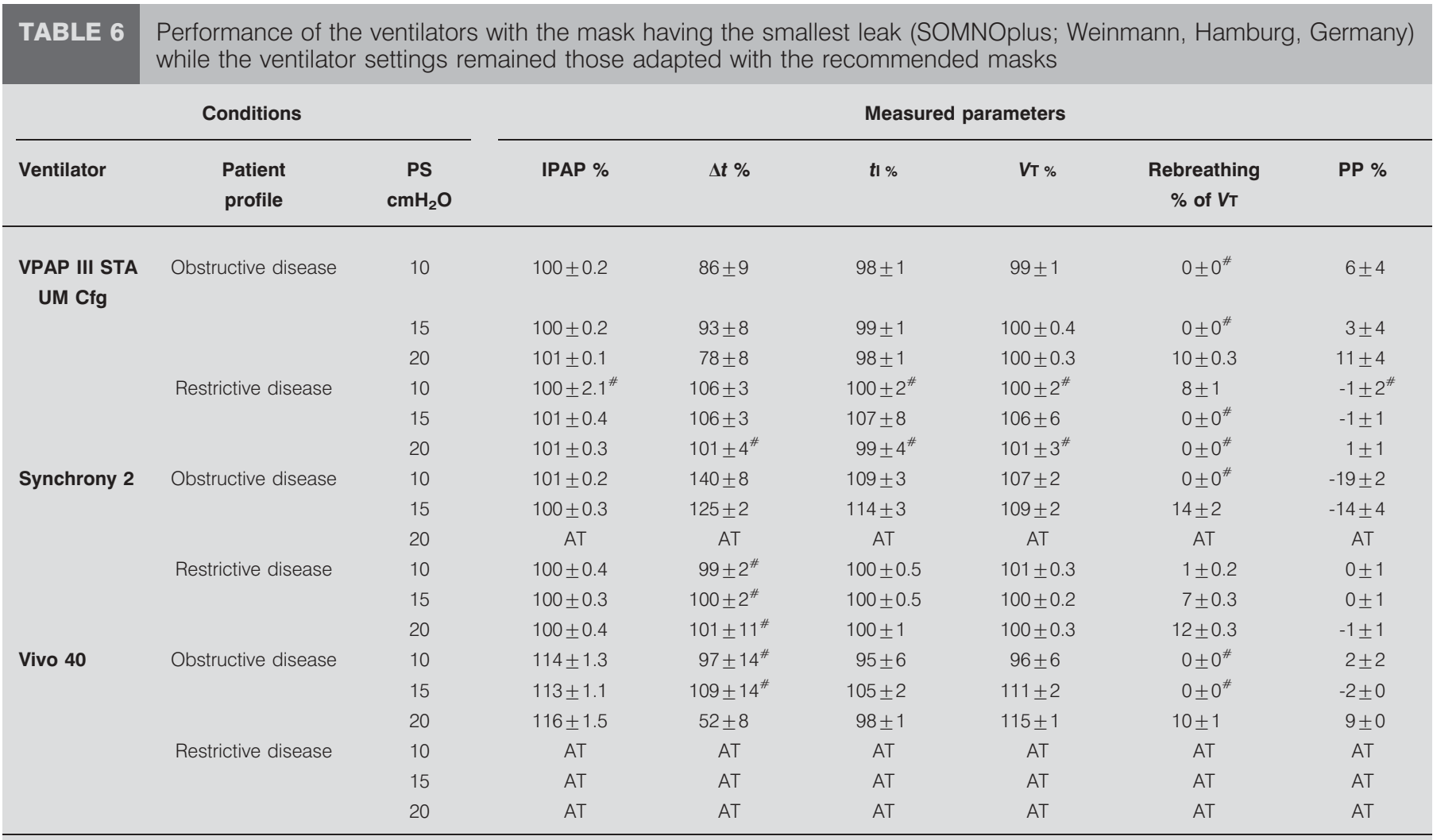

Data are presented as mean $\pm \mathrm{SD}$, unless otherwise stated. Values are the percentage of baseline conditions with the recommended mask (table 3), except for rebreathing, which is the percentage of tidal volume $(\mathrm{V} T)$, and pressurisation performance (PP), which is the percentage change versus baseline. PS: pressure support; IPAP: inspiratory positive airway pressure measured; $\Delta t$ : time from inspiration onset to airway pressure above positive end-expiratory pressure; $t$ : inspiratory time; $V T$ : tidal volume; rebreathing: percentage of expired volume remaining in the device circuit at the end of expiration; PP: pressurisation performance; AT: auto-triggering. VPAP III STA UM Cfg: ResMed, Saint Priest, France; Synchrony 2: Respironics, Nantes, Fracne); Vivo 40: Breas, Saint Priest). "\# : not statistically different from the baseline values.

having an acute respiratory failure, who could not breathe spontaneously for $>15 \mathrm{~min}$ [7]. These data suggest that the leak should exceed $20 \mathrm{~L} \cdot \mathrm{min}^{-1}$ when treating chronic respiratory failure and $22 \mathrm{~L} \cdot \mathrm{min}^{-1}$ in case of acute respiratory failure if the clinician wants to be sure that no expired gas is forced back to the circuit upstream of the exhalation port, ready to be in part rebreathed at the next inspiration. In our study, this was not the case for all the masks when a minimal PEEP of $4 \mathrm{cmH}_{2} \mathrm{O}$ was applied (fig. 3). Notably the SOMNOplus mask would induce an additional rebreathing in almost any clinical condition considering that its leak was equal to $12.6 \mathrm{~L} \cdot \mathrm{min}^{-1}$ for a $4 \mathrm{cmH}_{2} \mathrm{O}$ pressure (fig. 3).

The size of the mask may also affect the rebreathing by modifying the apparatus dead space and one can suggest that facial masks markedly increased this value. However, SAATCI et al. [15] demonstrated that the exhalation port in the nasal bridge of face masks generates a beneficial flow path that decreases the apparatus dead space, but only if leak during the expiratory phase is sufficient. In this condition, they observed that the dynamic apparatus dead space of the different masks varied from only 35 to $60 \mathrm{~mL}$ while their static volume varied between 100 and $400 \mathrm{~mL}$ [15]. This reduction of apparatus dead space induced by accurately positioning the exhalation port to the mask could therefore be considered as a beneficial effect of using a single circuit system if, obviously, the leak during the expiratory phase is sufficient.

The change of leakage is not a unique factor which may influence the result when the mask changes and our experimental conditions did not cover all the hazards and difficulties observed during noninvasive ventilation. A mask and mouth leakage may greatly disturb the ventilator performances. In addition, the interaction of mask with the face may impact the geometry of the airflow route and therefore the interaction between the nasal mask and the face and the connecting tube influence the total circuit resistance [16]. In addition, we did not evaluate the influence of the airway route breathing between the nose and the mouth; but it is clear that a facial mask allows a reduction of the upper airway resistance by breathing through the mouth and bypassing the nasal resistances which may represent $50 \%$ of the total airway resistances. This mouth route is generally preferred during acute respiratory failure [17]. To limit our evaluation to the MIML effects we used the same connecting tubes in each condition and according to WINDISCH et al. [16], we assumed a minor effect of the size of the mask on the total circuit resistance with the respect of its own resistance.

Our study had other limitations: $t \mathrm{I} / \mathrm{t}$ tot is generally shortened in a chronic obstructive pulmonary disease population in order 
TABLE 7 In vivo performance of the ventilators with the recommended masks condition

\begin{tabular}{|c|c|c|c|c|c|c|c|c|c|}
\hline Ventilator & $\begin{array}{l}\text { Recommended } \\
\text { mask }\end{array}$ & Subject & $\begin{array}{l}\text { PEEP } \\
\mathrm{cmH}_{2} \mathrm{O}\end{array}$ & $\begin{array}{l}\text { Peak P } \\
\mathrm{cmH}_{2} \mathrm{O}\end{array}$ & $\Delta t \mathrm{~ms}$ & $t \mid s$ & VT $\mathrm{mL}$ & $\begin{array}{c}\text { Rebreathing } \\
\quad \% \text { of } V_{T}\end{array}$ & PP \% \\
\hline \multirow[t]{3}{*}{ VENTImotion } & SOMNOplus & 1 & 3.7 & 18.1 & 265 & 1.2 & 865 & 0 & 18 \\
\hline & & 2 & 3.7 & 18.4 & 120 & 1.1 & 919 & 0 & 46 \\
\hline & & & $3.6 \pm 0.1$ & $19.1 \pm 1.3$ & $185 \pm 60$ & $1.2 \pm 0.1$ & $1020 \pm 159$ & $9 \pm 15$ & $33 \pm 12$ \\
\hline \multirow[t]{5}{*}{ VPAP III STA } & Ultra Mirage Full & 1 & 2.9 & 18.6 & 75 & 1.8 & 1522 & 0 & 63 \\
\hline & Face Medium & 2 & 3.1 & 16.8 & 168 & 1.8 & 1164 & 0 & 44 \\
\hline & & 3 & 2.7 & 17.3 & 77 & 1.3 & 1160 & 0 & 62 \\
\hline & & 4 & 2.9 & 18.5 & 77 & 1.8 & 1517 & 0 & 63 \\
\hline & & & $2.9 \pm 0.2$ & $17.8 \pm 0.9$ & $99 \pm 46$ & $1.7 \pm 0.3$ & $1341 \pm 206$ & 0 & $58 \pm 9$ \\
\hline
\end{tabular}

Data are presented as mean \pm SD, unless otherwise stated. PEEP: positive end-expiratory pressure; Peak P: peak airway pressure; $\Delta t$ : time from inspiration onset to airway pressure above positive end-expiratory pressure; tl: inspiratory time; VT: tidal volume; rebreathing: percentage of expired volume remaining in the device circuit at the end of expiration; PP: pressurisation performance. VENTImotion: Weinmann, Hamburg, Germany; VPAP III STA: ResMed, Saint Priest, France; SOMNOplus: Weinmann; Ultra Mirage Full Face Medium: ResMed.

to prolong $t \mathrm{E}$ and avoid intrinsic PEEP due to the expiratory flow limitation induced by the dynamic airway collapse. Because, unfortunately, our model did not simulate this phenomenon we did not observe intrinsic PEEP and we did not need to prolong $t E$. Nevertheless, it was interesting to observe that a similar pattern of breathing without mechanical ventilation during the simulation of both obstructive disease and obstructive disease did not induce a similar inspiratory trigger when adding the assisted mechanical ventilation.

The reason for which the Vivo 40 ventilator presented autotriggering with the recommended mask when simulating restrictive disease and when pressure support was $>10 \mathrm{cmH}_{2} \mathrm{O}$ is unclear. One explanation could be the presence of the pressure signal noise induced by both the low compliance of the simulated patient and the high pressure variation between inspiration and expiration which both facilitated auto-triggering.

The correction of the trigger sensitivity after mask change was made only in case of auto-triggering considering that if autotriggering does not occur; the clinician would not be able to clinically detect the change of trigger sensitivity and therefore would have no reason to change it.

TABLE 8 In vivo performance of the ventilators with the unrecommended masks

\begin{tabular}{|c|c|c|c|c|c|c|c|c|c|}
\hline \multicolumn{2}{|c|}{ Conditions } & \multicolumn{8}{|c|}{ Measurements } \\
\hline Ventilator & $\begin{array}{l}\text { Unrecommended } \\
\text { mask }\end{array}$ & Subject & PEEP \% & Peak P \% & $\Delta t \%$ & $t 1 \%$ & VT \% & $\begin{array}{c}\text { Rebreathing } \\
\% \text { of } V_{\top}\end{array}$ & PP \% \\
\hline \multirow[t]{3}{*}{ VENTImotion } & SOMNOplus & 1 & AT & AT & AT & AT & AT & AT & AT \\
\hline & & 2 & AT & AT & AT & AT & AT & AT & AT \\
\hline & & & AT & AT & AT & AT & AT & AT & AT \\
\hline \multirow[t]{5}{*}{ VPAP III STA } & Ultra Mirage Full & 1 & 105 & 107 & 107 & 83 & 86 & 39 & 63 \\
\hline & Face Medium & 2 & 112 & 109 & 108 & 104 & 102 & 0 & 44 \\
\hline & & 3 & 121 & 117 & 114 & 113 & 123 & 39 & 62 \\
\hline & & 4 & 110 & 98 & 148 & 85 & 77 & 30 & 63 \\
\hline & & & $112 \pm 6$ & $108 \pm 8$ & $119 \pm 20$ & $96 \pm 14$ & $97 \pm 20$ & $27 \pm 19$ & $-3 \pm 3$ \\
\hline
\end{tabular}

Data are presented as mean $\pm \mathrm{SD}$, unless otherwise stated. Values are the percentage of baseline conditions with the recommended mask, except for rebreathing, which is the percentage of tidal volume (VT), and pressurisation performance (PP), which is the percentage change versus baseline. PEEP: positive end-expiratory pressure; Peak P: peak airway pressure; $\Delta t$ : time from inspiration onset to airway pressure above positive end-expiratory pressure; $t \mathrm{l}$ : inspiratory time; rebreathing: percentage of expired volume remaining in the device circuit at the end of expiration; AT: auto-triggering. VENTImotion: Weinmann, Hamburg, Germany; VPAP III STA: ResMed, Saint Priest, France; SOMNOplus: Weinmann; Ultra Mirage Full Face Medium: ResMed 
In conclusion, changes in MIML through the mask exhalation port may modify patient-ventilator synchronisation and ventilator performance. Therefore, we recommend a routine clinical evaluation at every mask change in order to check inspiratory-trigger sensitivity and pressurisation, which should be adjusted if needed, and to check the absence of rebreathing. In addition our results and the pattern of breathing of patients under pressure support in the literature suggest that the leak of the mask should exceed $22 \mathrm{~L} \cdot \mathrm{min}^{-1}$, which was not observed with all the masks when the PEEP level was at its lowest value.

\section{SUPPORT STATEMENT}

The study was supported by the Association d'Entraide des Polios et Handicapés (ADEP; Cenon, France). B. Fauroux was supported by the Association Française contre les Myopathies (AFM ; Evry, France), ADEP, Assistance Publique-Hôpitaux de Paris (Paris, France), INSERM (Créteil, France), Legs Poix (Chancelerie des Universités de Paris, Paris) and Université Pierre et Marie Curie-Paris 6 (Paris).

\section{STATEMENT OF INTEREST}

None declared.

\section{REFERENCES}

1 Mehta S, Hill NS. Noninvasive ventilation. Am J Respir Crit Care Med 2001; 163: 540-577.

2 Schonhofer B, Sortor-Leger S. Equipment needs for noninvasive mechanical ventilation. Eur Respir J 2002; 20: 1029-1036.

3 Ferguson GT, Gilmartin M. $\mathrm{CO}_{2}$ rebreathing during BiPAP ventilatory assistance. Am J Respir Crit Care Med 1995; 151: 1126-1135.

4 Lofaso F, Brochard L, Touchard D, et al. Evaluation of carbon dioxide rebreathing during pressure support ventilation with airway management system (BiPAP) devices. Chest 1995; 108: 772-778.

5 Fauroux B, Leroux K, Desmarais G, et al. Performance of ventilators for noninvasive positive-pressure ventilation in children. Eur Respir J 2008; 31: 1300-1307.
6 Richard JC, Carlucci A, Breton L, et al. Bench testing of pressure support ventilation with three different generations of ventilators. Intensive Care Med 2002; 28: 1049-1057.

7 Lofaso F, Brochard L, Hang T, et al. Home versus intensive care pressure support devices. Experimental and clinical comparison. Am J Respir Crit Care Med 1996; 153: 1591-1599.

8 Miyoshi E, Fujino Y, Uchiyama A, et al. Effects of gas leak on triggering function, humidification, and inspiratory oxygen fraction during noninvasive positive airway pressure ventilation. Chest 2005; 128: 3691-3698.

9 Vignaux L, Tassaux D, Jolliet P. Performance of noninvasive ventilation modes on ICU ventilators during pressure support: a bench model study. Intensive Care Med 2007; 33: 1444-1451.

10 Stell IM, Paul G, Lee KC, et al. Noninvasive ventilator triggering in chronic obstructive pulmonary disease. A test lung comparison. Am J Respir Crit Care Med 2001; 164: 2092-2097.

11 Mehta S, McCool FD, Hill NS. Leak compensation in positive pressure ventilators: a lung model study. Eur Respir J 2001; 17: 259-267.

12 Borel JC, Sabil AK, Janssens JP, et al. Intentional leaks in industrial masks have a significant impact on efficacy of bilevel noninvasive ventilation: a bench test study. Chest 2009; 135: 669-677.

13 Fanfulla F, Delmastro M, Berardinelli A, et al. Effects of different ventilator settings on sleep and inspiratory effort in patients with neuromuscular disease. Am J Respir Crit Care Med 2005; 172: 619-624.

14 Navalesi P, Fanfulla F, Frigerio P, et al. Physiologic evaluation of noninvasive mechanical ventilation delivered with three types of masks in patients with chronic hypercapnic respiratory failure. Crit Care Med 2000; 28: 1785-1790.

15 Saatci E, Miller DM, Stell IM, et al. Dynamic dead space in face masks used with noninvasive ventilators: a lung model study. Eur Respir J 2004; 23: 129-135.

16 Windisch W, Buchholt A, Stahl CA, et al. Flow-dependent resistance of nasal masks used for non-invasive positive pressure ventilation. Respirology 2006; 11: 471-476.

17 Brochard L, Mancebo J, Wysocki M, et al. Noninvasive ventilation for acute exacerbations of chronic obstructive pulmonary disease. N Engl J Med 1995; 333: 817-822. 